\title{
PARABOLIC PARTIAL DIFFERENTIAL EQUATIONS WITH UNIFORMLY CONTINUOUS COEFFICIENTS
}

\author{
BY E. B. FABES AND N. M. RIVIÈRE
}

Communicated by A. Zygmund, July 22, 1965

Consider the following system of equations:

(1) $\sum_{j=1}^{M} \sum_{|\alpha| \leqq m} a_{\alpha}^{k, j}(x, t)(\partial / \partial x)^{\alpha} u_{j}-\delta_{k j}(\partial / \partial t) u_{k}=f_{k}, \quad k=1, \cdots, M$ here $x$ is a point in $E^{n}$ and $t \in(0, R), R<\infty$.

Assume the system (1) is parabolic in the sense of I. G. Petrovsky, i.e. the roots $\lambda(x, t ; z)$ of the equation:

$$
\operatorname{Det}\left(\sum_{|\alpha|=m} a_{\alpha}^{k, j}(x, t)(i z)^{\alpha}-\delta_{k j} \lambda\right)=0
$$

satisfy $\operatorname{Re}(\lambda(x, t ; z))<-\delta<0$ for $|z|=1$, independent of $(x, t)$.

Define $L_{0}^{p, m, 1}\left(E^{n} x(0, R)\right)$ to be the closure in the class of distributions of $E^{n+1}$, of the functions $u \in C_{0}^{\infty}\left(E^{n} x(0, \infty)\right)$ with respect to the norm:

$$
\begin{aligned}
\|u\|_{m, 1}= & \sum_{|\alpha| \leq m}\left(\int_{0}^{R} \int_{E^{n}}\left|(\partial / \partial x)^{\alpha} u\right|^{p} d x d t\right)^{1 / p} \\
& +\left(\int_{0}^{R} \int_{E^{n}}|\partial / \partial t|^{p} d x d t\right)^{1 / p} .
\end{aligned}
$$

Define $\left(L_{0}^{p, m, 1}\right)^{M}$ to be all vectors $u=\left(u_{1}, \cdots, u_{M}\right)$ with $u_{k} \in L_{0}^{p, m, 1}\left(E^{n} x(0, R)\right)$.

Concerning the coefficients of (1), assume that:

(i) $a_{\alpha}^{k, j}(x, t)$ are bounded and measurable over $E^{n} x(0, R)$ for all $\alpha, k, j$,

(ii) for $|\alpha|=m, a_{\alpha}^{k, j}(x, t)$ are uniformly continuous in $E^{n} x(0, R)$, for all $k, j$.

TheOREM. Given any vector-valued $f=\left(f_{1}, \cdots, f_{M}\right)$, where $f_{k}$ $\in L^{p}\left(E^{n} x(0, R)\right)$, there exists a unique $u \in\left(L_{0}^{p, m, 1}\right)^{M}$ satisfying system (1).

The proof of this theorem is based upon the following representation of the operator $L:\left(L_{0}^{p, m, 1}\right)^{M} \rightarrow\left(L^{p}\right)^{M}$ given by (1):

$$
L u=(I+K)\left((-1)^{m / 2} \Delta^{m / 2}+\partial / \partial t\right) I u,
$$


where $I$ is the identity matrix;

$$
\left((-1)^{m / 2} \Delta^{m / 2}+\partial / \partial t\right) I u=\left((-1)^{m / 2} \Delta^{m / 2} u_{j}+(\partial / \partial t) u_{j}\right),
$$

and

$$
\Delta u_{j}=\sum_{k=1}^{M}\left(\partial^{2} / \partial x_{k}^{2}\right) u_{j}
$$

$m$ is assumed to be an even number, and finally $K=K_{1}+K_{2}, K_{i}$ $=\left(K_{i}^{k, j}(x, t ; y, s)\right)$ where

$$
\begin{aligned}
K_{i}^{k, j}(u)=\lim _{\epsilon \rightarrow 0} \int_{0}^{t-\epsilon} \int_{E^{n}} K_{i}^{k, j}(x, t ; x-y, t-s) u(y, s) d y d s, & \\
& \text { for } u \in L^{p}\left(E^{n} x(0, R)\right) .
\end{aligned}
$$

Here $K_{1}$ is a matrix of singular integral operators, as defined in Abstract 65T-69, Notices Amer. Math. Soc. 12 (1965); while:

$$
\left\|K_{2}\right\|_{1}=\operatorname{ess} \sup _{(x, t)} \sum_{E^{n} x(0, R)} \int_{k, j}^{R} \int_{E^{n}}\left|K_{2}^{k, j}(x, t ; y, s)\right| d y d s<\infty .
$$

Therefore $I+K$ is a bounded operator from $\left(L^{p}\left(E^{n} x(0, R)\right)\right)^{M}$ into itself.

The proof consists in showing that $I+K$ is actually an isomorphism from $\left(L^{p}\left(E^{n} x(0, R)\right)\right)^{M}$ onto $\left(L^{p}\left(E^{n} x(0, R)\right)\right)^{M}$ and hence the problem is reduced to studying the operator $\left((-1)^{m / 2} \Delta^{m / 2}+\partial / \partial t\right) I$.

The theorem establishes existence and uniqueness for generalized solutions with initial condition zero. For the general initial value problem, the decomposition (2) reduces the problem to the operator $\left((-1)^{m / 2} \Delta^{m / 2}+\partial / \partial t\right) I$, with the same initial condition.

University of ChICAGo 